\title{
General Approach to Functional Forms for the Exponential Quadratic Operators in Coordinate-Momentum Space
}

\author{
Xiang-bin Wang [, C.H. Oh [ and L.C. Kwek [ \\ Department of Physics, Faculty of Science, \\ National University of Singapore, Lower Kent Ridge, \\ Singapore 119260, Republic of Singapore.
}

\begin{abstract}
In a recent paper [Nieto M M 1996 Quantum and Semiclassical Optics, 8 1061; quant-ph/9605032, the one dimensional squeezed and harmonic oscillator time-displacement operators were reordered in coordinate-momentum space. In this paper, we give a general approach for reordering multi-dimensional exponential quadratic operator(EQO) in coordinate-momentum space. An explicit computational formula is provided and applied to the single mode and double-mode EQO through the squeezed operator and the time displacement operator of the harmonic oscillator.
\end{abstract}

\footnotetext{
${ }^{1}$ E-mail address: scip7236@leonis.nus.edu.sg

${ }^{2}$ E-mail address: phyohch@leonis.nus.edu.sg

${ }^{3}$ E-mail address: scip6051@leonis.nus.edu.sg
} 


\section{Introduction}

The exponential quadratic operator(EQO) plays an important role in quantum mechanics and quantum optics. In quantum optics, such operators occur ubiquitously in topics related to coherent and squeezed states. Consequently, it has always been important to devise and explore simplifying computational procedures for reducing these operators into some manageable forms. In many applications, one usually expresses these operators into their normal ordered forms. In a recent paper[1], it has been shown that it is also convenient to consider the reordering of these operators in coordinate-momentum $(x-p)$ phase space as:

$$
\exp [\delta] \exp \left[\alpha x^{2}\right] \exp [\beta x \partial] \exp \left[\gamma \partial^{2}\right]
$$

where $\delta, \alpha, \beta$ and $\gamma$ are c-number parameters. Such reorderings, together with the following identities $[1,2]$ :

$$
\begin{gathered}
\exp [c \partial] h(x)=h(x+c) \\
\exp [\tau x \partial] h(x)=h\left(x e^{\tau}\right) \\
\exp \left[c \partial^{2}\right] h(x)=\frac{1}{[4 \pi c]^{1 / 2}} \int_{-\infty}^{\infty} \exp \left[-\frac{(y-x)^{2}}{4 c}\right] h(y) d y
\end{gathered}
$$

facilitate the computations of the wavefunction. Moreover, as pointed out in ref[1], reordering the operators in $x-p$ space can be applied to systems[3] with time dependent potentials such as:

$$
V(x, t)=g^{(2)}(t) x^{2}+g^{(1)}(t) x+g^{(0)}(t)
$$

Reordering EQO in $x-p$ phase space is therefore an interesting problem that deserves further investigations. Following Wei and Norman[4], Nieto[1] has reduced the one dimensional EQO reordering problem in $x-p$ space into the solution of four coupled first-order differential equations with four unknowns. However, a direct calculation formula that relates the EQO to its reordered form is not available. Furthermore, the results have not been extended to the $n$-dimensional case. 
In this paper, we start within the framework of $x$ - $p$ space and construct a very general approach which is suitable to reordering arbitrary mode EQOs to its reordered form in $x-p$ space. In the following section, we will outline this general approach and summarize the essential steps. In section 3, we show that this general approach yields an explicit formula for the reordering of arbitrary one-dimensional EQO. The formula is then applied to the one dimensional squeezed operator and time-displacement operator of the harmonic oscillator. The results are the same as ref[1], but unlike ref[1], we need not solve a system of coupled differential equations. Finally, in section 4, we consider the reordering of EQO in two dimensions and apply the same technique to the two-dimensional squeezed operator and time displacement operator of the coupled harmonic oscillator.

\section{General Approach}

We denote the $n$-dimensional coordinate and momentum operators as:

$$
x=\left(x_{1}, x_{2} \cdots x_{n}\right) \quad ; \quad \partial=i p=\left(\partial_{1}, \partial_{2}, \cdots \partial_{n}\right)
$$

The commutation rule for these operators is

$$
\left[x_{i}, \partial_{j}\right]=-\delta_{i j}
$$

Without any loss of generality, we shall consider the following EQO,

$$
U=\exp \left[\frac{1}{2}(x, \partial)\left(\begin{array}{cc}
D_{1} & F \\
\widetilde{F} & D_{2}
\end{array}\right)\left(\begin{array}{l}
\widetilde{x} \\
\widetilde{\partial}
\end{array}\right)\right]
$$

where $D_{1}, D_{2}, F$ are $n \times n$ complex matrices and $D_{1}=\widetilde{D_{1}}$ and $D_{2}=\widetilde{D_{2}}$; the tilde sign denotes the transpose of a matrix. It is convenient to introduce the symmetric matrix $R$

as $\left(\begin{array}{cc}D_{1} & F \\ \widetilde{F} & D_{2}\end{array}\right)$ and operator $\hat{A} \equiv \frac{1}{2}(x, \partial) R\left(\begin{array}{c}\widetilde{x} \\ \widetilde{\partial}\end{array}\right)$. By direct calculations, we first note that if $L$ and $M$ are $n \times n$ complex matrices and $N$ is a symmetric $n \times n$ complex matrix, then the following identities hold:

$$
\left[\frac{1}{2} x N \widetilde{x}, \partial M\right]=\frac{1}{2}(x N \widetilde{x} \partial M-\partial M x N \widetilde{x})=-x \cdot N M
$$




$$
\begin{array}{ccc}
{\left[\frac{1}{2} \partial N \widetilde{\partial}, x M\right]=\frac{1}{2}(\partial N \widetilde{\partial} x M} & -x M \partial N \widetilde{\partial})= & \partial \cdot N M \\
{[x L \widetilde{\partial}, x M]} & = & x \cdot L M \\
{[x L \widetilde{\partial}, \partial M]} & = & -\partial \cdot \widetilde{L} M .
\end{array}
$$

From the above identities, one arrives at

$$
[\hat{A},(x, \partial) K]=(x, \partial) R \Sigma^{-1} K
$$

where $K$ is an arbitrary $2 n \times 2 n$ complex matrix and $\Sigma$ denotes $\left(\begin{array}{cc}0 & I \\ -I & 0\end{array}\right)$ with $I$ as an $n \times n$ identity matrix.

Using the above formulae and commutation relations, one can recursively compute the following relations

$$
\begin{aligned}
{[\hat{A},(x, \partial)] } & =(x, \partial) R \Sigma^{-1} \\
{[\hat{A},[\hat{A},(x, \partial)]] } & =(x, \partial) R \Sigma^{-1} \cdot R \Sigma^{-1}=(x, \partial)\left(R \Sigma^{-1}\right)^{2} \\
& \ldots
\end{aligned}
$$

Applying Baker-Campbell-Hausdorff (BCH) relations, one gets

$$
U(x, \partial) U^{-1}=(x, \partial)+[\hat{A},(x, \partial)]+\frac{1}{2 !}[\hat{A},[\hat{A},(x, \partial)]]+\cdots
$$

which immediately yields

$$
U(x, \partial) U^{-1}=(x, \partial) \cdot \exp \left(R \cdot \Sigma^{-1}\right)
$$

We next denote $T=\left(\begin{array}{ll}T_{11} & T_{12} \\ T_{21} & T_{22}\end{array}\right)=\exp \left(R \cdot \Sigma^{-1}\right)$ then we have

$$
U(x, \partial) U^{-1}=(x, \partial) \cdot\left(\begin{array}{ll}
T_{11} & T_{12} \\
T_{21} & T_{22}
\end{array}\right)
$$

where $T_{11}, T_{12}, T_{21}$ and $T_{22}$ are $n \times n$ matrices. The $T_{i j}, i, j=1,2$ matrices are not independent. To see this, we note that the exponential matrix, $\exp \left(R \Sigma^{-1}\right)$, satisfies:

$$
\Sigma^{-1} \exp \left(-R \Sigma^{-1}\right) \Sigma=\exp \left(-\Sigma^{-1} R\right)=\exp \left(R \Sigma^{-1}\right) .
$$


As $\Sigma^{-1}=-\Sigma$, the above equation (13) becomes

$$
\exp \left(\overparen{\curvearrowright} \Sigma^{-1}\right) \Sigma \exp \left[\left(R \Sigma^{-1}\right)\right)=\Sigma
$$

which, in our notation, can be recast as

$$
\widetilde{T} \Sigma T=\Sigma .
$$

Expanding and equating the entries in eq(15), we get

$$
\begin{aligned}
\widetilde{T}_{22} T_{11}-\widetilde{T}_{12} T_{21} & =1 ; \\
\widetilde{T}_{21} T_{11} & =\widetilde{T}_{11} T_{21} ; \\
\widetilde{T}_{22} T_{12} & =\widetilde{T}_{12} T_{22} .
\end{aligned}
$$

One can then easily manipulate eq(16) to get the relation

$$
T_{11}=\widetilde{T}_{22}^{-1}+T_{12} T_{22}^{-1} T_{21}
$$

Furthermore, by these identities, one can always have the following decomposition

$$
\left(\begin{array}{ll}
T_{11} & T_{12} \\
T_{21} & T_{22}
\end{array}\right)=\left(\begin{array}{cc}
I & W \\
0 & I
\end{array}\right)\left(\begin{array}{cc}
e^{Y} & 0 \\
0 & e^{-\widetilde{Y}}
\end{array}\right)\left(\begin{array}{cc}
I & 0 \\
Z & I
\end{array}\right)
$$

with

$$
W=T_{12} T_{22}{ }^{-1}, Z=T_{22}{ }^{-1} T_{21}, Y=-\ln \widetilde{T_{22}} .
$$

Let

$$
\begin{aligned}
& U_{1}=\exp \left[\frac{1}{2}(x, \partial)\left(\begin{array}{cc}
-W & 0 \\
0 & 0
\end{array}\right)\left(\begin{array}{l}
\widetilde{x} \\
\widetilde{\partial}
\end{array}\right)\right] \\
& U_{2}=\exp \left[\frac{1}{2}(x, \partial)\left(\begin{array}{cc}
0 & Y \\
\widetilde{Y} & 0
\end{array}\right)\left(\begin{array}{l}
\widetilde{x} \\
\widetilde{\partial}
\end{array}\right)\right],
\end{aligned}
$$

and

$$
U_{3}=\exp \left[\frac{1}{2}(x, \partial)\left(\begin{array}{ll}
0 & 0 \\
0 & Z
\end{array}\right)\left(\begin{array}{l}
\widetilde{x} \\
\widetilde{\partial}
\end{array}\right)\right] .
$$


Using eq(11), one has

$$
\begin{aligned}
U_{1}(x, \partial) U_{1}^{-1} & =(x, \partial)\left(\begin{array}{cc}
I & W \\
0 & I
\end{array}\right), \\
U_{2}(x, \partial) U_{2}^{-1} & =(x, \partial)\left(\begin{array}{cc}
e^{Y} & 0 \\
0 & e^{-\widetilde{Y}}
\end{array}\right), \\
U_{3}(x, \partial) U_{3}^{-1} & =(x, \partial)\left(\begin{array}{cc}
I & 0 \\
Z & I
\end{array}\right)
\end{aligned}
$$

Thus the reordered EQO, $U^{\prime}=U_{1} U_{2} U_{3}$, satisfies the following relation

$$
U^{\prime}(x, \partial) U^{\prime-1}=(x, \partial)\left(\begin{array}{cc}
I & W \\
0 & I
\end{array}\right)\left(\begin{array}{cc}
e^{Y} & 0 \\
0 & e^{-\widetilde{Y}}
\end{array}\right)\left(\begin{array}{cc}
I & 0 \\
Z & I
\end{array}\right)=(x, \partial)\left(\begin{array}{cc}
T_{11} & T_{12} \\
T_{21} & T_{22}
\end{array}\right) .
$$

As shown in the Appendix, operator $U^{-1} U^{\prime}$ commutes with all $x_{i}$ and $p_{i}$ so that $U$ differs from $U^{\prime}$ by a c-number factor. This factor can be shown to be unity by evaluating the matrix element between any two states to $U$ and $U^{\prime}$ respectively (see Appendix or ref[5] for details). Finally, we arrive at the following formula for reordering the EQOs in an $n$-dimensional $x$ - $p$ space:

$$
\exp \left[\frac{1}{2}(x, \partial) R\left(\begin{array}{c}
\widetilde{x} \\
\widetilde{\partial}
\end{array}\right)\right]=e^{\frac{1}{2}(\operatorname{tr} Y)} e^{-\frac{1}{2} x W \widetilde{x}} e^{x Y \widetilde{\partial}} e^{\frac{1}{2} \partial Z \widetilde{\partial}}
$$

In principle, one can reorder any $n$-dimensional EQO in x-p space through eq(21).

In summary, one can compute the EQO reordering in $x-p$ phase space according to the following fixed procedure:

1. Given any EQO, one can rewrite it in the form of eq(6) to obtain the matrix $R$, and hence the matrices $D_{1}, D_{2}$ and $F$.

2. One then computes the exponential matrix $\exp \left(R \cdot \Sigma^{-1}\right)=\exp \left(\begin{array}{cc}F & -D_{1} \\ D_{2} & -\widetilde{F}\end{array}\right)$ and obtains the matrix $\left(\begin{array}{ll}T_{11} & T_{12} \\ T_{21} & T_{22}\end{array}\right)$. 
3. By eq(19), one can construct $W, Z$ and $Y$ explicitly.

4. Finally using eq(21), one arrives at the reordered form.

\section{One-Dimensional Application}

We now apply the above results to one-dimensional problems and the general procedure simplifies considerably in this case. For one dimensional problems, we have

$$
U=\exp \left[\frac{1}{2}(x, \partial)\left(\begin{array}{ll}
a & c \\
c & b
\end{array}\right)\left(\begin{array}{l}
x \\
\partial
\end{array}\right)\right]
$$

where $a, b$ and $c$ are all arbitrary c-numbers. Straightforwardly, we easily get

$$
\begin{aligned}
\exp \left(R \cdot \Sigma^{-1}\right)=\exp \left(\begin{array}{ll}
c & -a \\
b & -c
\end{array}\right) & =\left(\begin{array}{cc}
\cosh \theta+c \cdot \sinh \theta / \theta & -a \cdot \sinh \theta / \theta \\
b \cdot \sinh \theta / \theta & \cosh \theta-c \cdot \sinh \theta / \theta
\end{array}\right) \\
& =\left(\begin{array}{cc}
T_{11} & T_{12} \\
T_{21} & T_{22}
\end{array}\right)
\end{aligned}
$$

where $\theta=\sqrt{c^{2}-a b}$. Using eq(19), one obtains

$W=\frac{-a}{\theta} \sinh \theta \cdot\left(\cosh \theta-\frac{c}{\theta}\right)^{-1} ; Y=-\ln \left[\cosh \theta-\frac{c}{\theta} \sinh \theta\right] ; Z=\frac{b}{\theta} \sinh \theta \cdot\left(\cosh \theta-\frac{c}{\theta}\right)^{-1}$.

Substituting eq(25) into eq(21) gives

$$
\begin{aligned}
& \exp \left[\frac{1}{2}(x, \partial)\left(\begin{array}{ll}
a & c \\
c & b
\end{array}\right)\left(\begin{array}{l}
x \\
\partial
\end{array}\right)\right]=\frac{1}{\sqrt{\cosh \theta-\frac{c}{\theta}}} \cdot \\
& \exp \left[\frac{1}{2} \frac{a}{\theta} \sinh \theta\left(\cosh \theta-\frac{c}{\theta}\right)^{-1} x^{2}\right] \exp \left[-\ln \left(\cosh \theta-\frac{c}{\theta}\right) x \partial\right] \exp \left[\frac{1}{2} \frac{b}{\theta} \sinh \theta\left(\cosh \theta-\frac{c}{\theta}\right)^{-1} \partial^{2}\right]
\end{aligned}
$$

$\mathrm{Eq}(26)$ is an explicit formula for reordering any arbitrary one-dimensional EQO.

To illustrate the use of eq(26), we consider two specific examples[1]: the time displacment operator of the harmonic oscillator and the squeezed operator in one dimension. For time displacement operator of the harmonic oscillator,

$$
T=\exp \left[\frac{-i t}{2}\left(x^{2}-\partial^{2}\right)\right]
$$


Comparing this expression with eq(26), we get

$$
a=-i t ; b=i t ; c=0 ; \theta=\sqrt{0^{2}-(-i t) \cdot i t}=i t
$$

Using eq(26), it follows

$$
T=\frac{1}{\sqrt{\cos t}} \exp \left[-\frac{i}{2} \tan t x^{2}\right] \exp [-\ln \cos t x \partial] \exp \left[\frac{i}{2} \tan t \partial^{2}\right],
$$

which is just eq(44) of ref.[1].

The one dimensional squeezed operator is (eq(9) of ref.[1]):

$$
S(z)=\exp \left[-z_{1}(x \partial+1 / 2)+i z_{2}\left(x^{2}+\partial\right) / 2\right]
$$

which can be rewritten as

$$
U=\exp \left[\frac{1}{2}(x, \partial)\left(\begin{array}{cc}
i z_{2} & -z_{1} \\
-z_{1} & i z_{2}
\end{array}\right)\left(\begin{array}{l}
x \\
\partial
\end{array}\right)\right] .
$$

Comparing it with eq(12),

$$
a=b=i z_{2}, c=-z_{1}, \theta=\sqrt{z_{1}^{2}+z_{2}^{2}}=r
$$

Using eq(26), one easily sees that

$$
\begin{gathered}
U=\frac{1}{\sqrt{\cosh r+\frac{z_{1}}{r} \sinh r}} \cdot \exp \left[\frac{i z_{2}}{2 r} \sinh r\left(\cosh r+\frac{z_{1}}{r} \sinh r\right)^{-1} x^{2}\right] . \\
\exp \left[-\ln \left(\cosh r+\frac{z_{1}}{r} \sinh r\right) x \partial\right] \exp \left[\frac{i z_{2}}{2 r} \sinh r\left(\cosh r+\frac{z_{1}}{r} \sinh r\right)^{-1} \partial^{2}\right],
\end{gathered}
$$

which is just the eqs.(37) of ref.[1].

\section{Two-Dimensional Application}

Finally, we consider the two dimensional problem and reorder some two dimensional EQOs in $x$ - $p$ space. The two-mode squeezed operator is given by $[8]$

$$
S=\exp \left[g a_{1} a_{2}-g^{*} a_{1}{ }^{+} a_{2}{ }^{+}\right]
$$


Using $\left(a_{i}{ }^{+}, a_{i}\right)=\frac{1}{\sqrt{2}}\left(x_{i}, \partial_{i}\right)\left(\begin{array}{cc}1 & 1 \\ -1 & 1\end{array}\right)$, we can rewrite this squeezed operator $S$ as

$$
S=\exp \left[\frac{1}{2}(x, \partial) N\left(\begin{array}{cc}
\left(-g^{*}\right) \sigma & 0 \\
0 & (g) \sigma
\end{array}\right) N^{-1}\left(\begin{array}{l}
\widetilde{x} \\
\widetilde{\partial}
\end{array}\right)\right]
$$

where $\sigma=\left(\begin{array}{ll}0 & 1 \\ 1 & 0\end{array}\right), N=\frac{1}{\sqrt{2}}\left(\begin{array}{cc}I & I \\ -I & I\end{array}\right)$ and $I$ is the $2 \times 2$ identity matrix. Here, both $x$ and $\partial$ are two-dimensional (two modes) vectors. Let $R$ be the matrix given by

$$
R=N\left(\begin{array}{cc}
-g^{*} \sigma & 0 \\
0 & g \sigma
\end{array}\right) N^{-1}
$$

It is easy to see that

$$
\begin{aligned}
\exp \left(R \Sigma^{-1}\right) & =N \cdot \exp \left(\begin{array}{cc}
0 & g^{*} \sigma \\
g \sigma & 0
\end{array}\right) \cdot N^{-1} \\
& =\left(\begin{array}{cc}
\cosh |g| \cdot I+\frac{g+g^{*}}{2|g|} \sinh |g| \cdot \sigma & \frac{g^{*}-g}{2|g|} \sinh |g| \cdot \sigma \\
\frac{g-g^{*}}{2|g|} \sinh |g| \cdot \sigma & \cosh |g| \cdot I-\frac{g+g^{*}}{2|g|} \sinh |g| \cdot \sigma
\end{array}\right)
\end{aligned}
$$

Following our general procedure and denoting $s_{ \pm}$as $\frac{g \pm g^{*}}{2|g|} \sinh |g|$, we get via eq(21)

$$
\left\{\begin{array}{c}
W=\frac{-s_{-}}{\cosh ^{2}|g|-s_{+}^{2}}\left(\begin{array}{cc}
s_{+} & \cosh |g| \\
\cosh |g| & s_{+}
\end{array}\right) \\
Z=\frac{s_{-}}{\cosh ^{2}|g|-s_{+}^{2}}\left(\begin{array}{cc}
s_{+} & \cosh |g| \\
\cosh |g| & s_{+}
\end{array}\right) \\
Y=-\ln \left(\begin{array}{cc}
\cosh |g| & -s_{+} \\
-s_{+} & \cosh |g|
\end{array}\right)
\end{array}\right.
$$

With these quantities solved, one gets from eq(11) the $x-p$ reordered form for the two modes squeezed state operator.

For the time displacement operator of a two-dimensional coupled harmonic oscillator with the Hamiltonian

$$
H=\frac{1}{2}(x \widetilde{x}+\partial \widetilde{\partial})+\lambda x_{1} x_{1}, \quad-1 \leq \lambda \leq 1
$$


we have the time displacement operator

$$
U=\exp \left[\frac{1}{2}(x, \partial) R\left(\begin{array}{l}
\widetilde{x} \\
\widetilde{\partial}
\end{array}\right)\right]
$$

where $R=\left(\begin{array}{cc}-i t M & 0 \\ 0 & i t I\end{array}\right)$ and $M=\left(\begin{array}{cc}1 & \lambda \\ \lambda & 1\end{array}\right)$. With these notations, we see that

$$
\exp \left(R \Sigma^{-1}\right)=\left(\begin{array}{cc}
\cos (t \sqrt{M}) & i \sqrt{M} \sin (t \sqrt{M}) \\
\frac{i \sin (t \sqrt{M})}{\sqrt{M}} & \cos (t \sqrt{M})
\end{array}\right)
$$

where $\sqrt{M}=\left(\begin{array}{cc}\cos \omega & \sin \omega \\ \sin \omega & \cos \omega\end{array}\right)$ and $\omega=\frac{1}{2} \sin ^{-1} \lambda$. Again using eq(9), we have

$$
\left\{\begin{array}{l}
W=i \sqrt{M} \tan (t \sqrt{M}) \\
Z=\frac{i}{\sqrt{M}} \tan (t \sqrt{M}) \\
Y=-\ln [\cos (t \sqrt{M})]
\end{array}\right.
$$

From eq(21), the $x$ - $p$ reordered form for the two-dimensional time displacement operator of the coupled harmonic oscillator can thus be written down.

\section{Appendix}

In this appendix, we shall show that the operator $U^{-1} U^{\prime}$ commutes with the position and momentum operators and consequently $U$ differs from $U^{\prime}$ by a c-number which can be shown to be unity. We first note that $U$ and $U^{\prime}$ satisfy the relation

$$
U(x, \partial) U^{-1}=U^{\prime}(x, \partial) U^{\prime-1}=(x, \partial) T .
$$

For the position operator, since

$$
U x U^{-1}=U^{\prime} x U^{\prime-1}
$$


we have

$$
x U^{-1} U^{\prime}=U^{-1} U^{\prime} x .
$$

This means $U U^{\prime-1}$ commutes with all position operators. Similarly, one can show that $U^{-1} U^{\prime}$ commutes all momentum operators. Clearly, by Schur's lemma, one concludes that $U^{-1} U^{\prime}$ is proportional to unity and thus $U^{\prime}=c \cdot U$.

We next determine the value of $c$. Let $\mid f>$ and $\mid g>$ be the eigenstate of operator $x$ and $\partial$ with zero eigenvalue. Clearly, $\langle f| x=0$ and $\partial|g\rangle=0$. Further, using the definitition of $U^{\prime}$, one can immediately see that

$$
\begin{aligned}
<f\left|U^{\prime}\right| g> & =<f\left|U_{1} U_{2} U_{3}\right| g> \\
= & <f\left|U_{2}\right| g>\text { since }<f \mid U_{1}=0 \text { and } U_{3} \mid g>=0 \\
= & <f\left|\exp \left(-\frac{1}{2} \partial \widetilde{Y} \widetilde{x}\right)\right| g> \\
= & <f\left|\exp \left(-\frac{1}{2}\{x Y \widetilde{\partial}+\operatorname{tr} \tilde{Y}\}\right)\right| g> \\
= & \exp \left(-\frac{1}{2} \operatorname{tr} \ln T_{22}\right)<f \mid g>.
\end{aligned}
$$

We denote $e^{t R \Sigma^{-1}}$ by the following form:

$$
e^{t R \Sigma^{-1}}=\left(\begin{array}{ll}
T_{11}(t) & T_{12}(t) \\
T_{21}(t) & T_{22(t)}
\end{array}\right)
$$

and proceed to calculate the matrix element value of $e^{t \hat{A}}$ between $\langle f|$ and $|g\rangle$ as

$$
v(t)=\left\langle f\left|e^{t \hat{A}}\right| g\right\rangle .
$$

However, we note that $\partial \mid g>=0$ and $<f \mid x=0$, so that the derivative $v^{\prime}(t)$ is given by

$$
v^{\prime}(t)=\frac{1}{2}\left\langle f\left|\left(\partial D_{2} \widetilde{\partial}+\operatorname{tr} F\right) e^{t \hat{A}}\right| g\right\rangle .
$$

In eq(38), we have used the identity $\partial F \widetilde{x}=x \widetilde{F} \widetilde{\partial}+\operatorname{tr} F$. From the transformation property of operator $e^{t \hat{A}}$ in eq(12) we get the following matrix identity :

$$
\begin{aligned}
0 & =\left\langle f\left|e^{t \hat{A}} \widetilde{\partial} \partial\right| g\right\rangle \\
& =\left\langle f\left|\left[\widetilde{T}_{12}(t) \widetilde{x}+\widetilde{T}_{22}(t) \widetilde{\partial}\right]\left[x T_{12} t+\widetilde{\partial} T_{22}(t)\right] e^{t \hat{A}}\right| g\right\rangle \\
& =\widetilde{T}_{22}(t) T_{12}(t) v(t)+\widetilde{T}_{22}(t)\left\langle f\left|\widetilde{\partial} \partial e^{t \hat{A}}\right| g\right\rangle T_{22}(t)
\end{aligned}
$$


Without loss of generality, one can assume that $\operatorname{det}\left(T_{22}(t)\right) \neq 0$, so that

$$
\left\langle 0 f\left|\widetilde{\partial} \partial e^{t \hat{A}}\right| g\right\rangle=-T_{12}(t) T_{22}(t)^{-1} v(t)
$$

which leads to

$$
\left\langle 0\left|\partial D_{2} \widetilde{\partial} e^{t \hat{A}}\right| 0\right\rangle=-v(t) \operatorname{tr}\left[D_{2} T_{12} T_{22}(t)^{-1}\right] .
$$

Substituting eq(43) into eq(38) we get

$$
v^{\prime}(t)=v(t) \frac{1}{2} \operatorname{tr}\left[\widetilde{F}-D_{2} T_{12} T_{22}(t)^{-1}\right] .
$$

On the other hand, one sees that the derivative of the $T$ matrix is given by

$$
\frac{d}{d t}\left(\begin{array}{ll}
T_{11}(t) & T_{12}(t) \\
T_{21}(t) & T_{22}(t)
\end{array}\right)=\left(\begin{array}{cc}
F & -D_{1} \\
D_{2} & -\widetilde{F}
\end{array}\right)\left(\begin{array}{ll}
T_{11}(t) & T_{12}(t) \\
T_{21}(t) & T_{22}(t)
\end{array}\right) .
$$

Immediately it follows

$$
\frac{d T_{22}(t)}{d t}=D_{2} T_{12}(t)-\widetilde{F} T_{22}(t)
$$

Putting eq(46) into eq(44), one sees that $v(t)$ satisfies the differential equation

$$
v^{\prime}(t)=-\frac{1}{2} v(t) \operatorname{tr}\left[\frac{d T_{22}(t)}{d t} T_{22}(t)^{-1}\right]
$$

which can be integrated using the condition $v(0)=<f \mid g>$ to give

$$
v(t)=\exp \left[-\frac{1}{2} \operatorname{tr} \ln T_{22}(t)\right]<f \mid g>
$$

Comparing eq(37) and eq(47) and remembering that $U=\mathrm{e}^{A}$, the value of $c$-number factor is unity so that $U=U^{\prime}$.

\section{References}

[1] Nieto M M 1996 Quantum and Semiclassical Optics 8 1061, quant-ph/9605032

[2] Nieto M M 1996 Phys. Lett. A219 180 
[3] Nieto M M and Truax D R 1997 J. Math. Phys. 38 84, quant-ph/9608008; Nieto M M and Truax D R 1997 J. Math. Phys. 38 98, quant-ph/9608009

[4] Wei J and Norman E 1963 J. Math. Phys. 4575

[5] Wang Xiang-bin, Yu Si-xia and Zhang Yong-de 1994 J. Phys. A: Math. Gen. 276563

[6] McCoy N H 1932 Proc. Edinburg Math. Soc3 118

[7] Wilcox R M 1967 J. Math. Phys.8 962

[8] Walls D F and Milburn G J 1994 Quantum Optics (Berlin:Springer-Verlag) p.22 